Ann. Biol. anim. Bioch. Biophys., 1977, 17 (5 B), 807-825.

\title{
Régulation de l'activité des enzymes de la lipolyse et de la lipogenèse dans le tissu adipeux
}

\author{
par Simonne ROUS
}

Département de Biochimie médicale, Université de Genève Rue Ecole de Médecine 20-1211 Genève 4 (Suisse).

Summary. Regulation of lipolytic and lipogenic enzyme activity in adipose tissue.

The fixation of hormones termed lipolytic on their receptors in adipose tissue membranes, with subsequent adenylcyclase activation and increase in intracellular cyclic AMP concentration, is a convenient way of explaining most of their lipase-stimulating effect. In fact, cyclic AMP fixes on the regulatory subunit of the kinase responsible for lipase phosphorylation, causing this subunit to detach from the catalytic subunit which may then phosphorylate the lipase and activate it.

Animal sensitivity to different lipolytic hormones varies, depending on whether there is a larger or smaller number of specific receptors of these hormones on the plasma membranes of their adipose tissue.

Glucocorticoids do not act as other lipolytic hormones by adenylcyclase activation, but by initiating the synthesis of this enzyme in the nucleus. Insulin antilipolytic action could be explained by activation of membrane $P$ diesterase, a recently discovered enzyme.

Enzymes responsible for fatty acid synthesis are controlled in two ways : by short-term regulation expressed solely by changes in enzyme activity, and by long-term regulation causing changes in enzyme concentration. Enzyme systems furnishing NADPH do not appear to play a role ; there seems to always be an excess of NADPH, as of acetyl CoA or ATP.

Short-term control mainly affects acetyl CoA carboxylase by long-chain acyl CoA. Even at physiological concentrations, these latter act as competitive inhibitors of acetyl CoA on its fixation site on acetyl CoA carboxylase. By inhibiting citrate diffusion outside the mitochondria, acyl CoA may cause cytoplasmic acetyl CoA deficiency, and the citrate itself may aggravate this deficiency by inhibiting pyruvate dehydrogenase.

Depending on the species, acetyl COA and acetoacetyl COA synthetases could play a particularly important regulating role in adipose tissue lipogenesis.

Lipolysis and lipogenesis are two closely related process. Insulin is a pre-eminently lipogenic hormone which inhibits the release of free fatty acids and favours longterm lipogenic regulation in the adipose tissue by stimulating the synthesis of acetyl CoA carboxylase and fatty acid synthetase as opposed to glucocorticoids which show an inhibitory effect.

\section{Régulation de la lipolyse.}

Le tissu adipeux de rat contient plusieurs enzymes lipolytiques (Jensen, 1971 ; Steinberg, 1972) qui diffèrent les unes des autres par leur $\mathrm{pH}$ optimum, leur substrat, leur localisation intracellulaire, la cinétique de leur réaction. 
Deux de ces enzymes sont principalement impliquées dans l'hydrolyse des triglycérides : la lipoprotéine lipase (LPL) qui n'intervient que sur les triglycérides faisant partie des molécules de lipoprotéines contenues dans le plasma ef qui contrôle donc la captation par le tissu adipeux des acides gras circulants ; la lipase hormonosensible (LHS) qui hydrolyse les triglycérides contenus dans le tissu adipeux en réponse aux hormones lipolytiques comme l'adrénaline, le glucagon, I'ACTH, etc... (VauGhan et Steinberg, 1965). Le pH optimum d'action de la LPL est de 8,4 , celui de la LHS est de 7 .

Il existe cependant dans le tissu adipeux d'autres enzymes qui, comme la LHS, font partie du groupe des lipases qui agissent sur les triglycérides renfermant des acides gras à longue chaîne mais qui, contrairement à la LHS, agissent à $\mathrm{pH}$ acide, et des triglycérides lipases qui hydrolysent les triglycérides renfermant des acides gras à petit nombre de carbones (Crum et Lech, 1969).

Malgré que la LHS soit capable d'hydrolyser successivement les 3 fonctions esters des triglycérides, on ignore cependant s'il existe une diglycéride lipase distincte (Boyer, 1975). Cependant, une monoglycéride lipase vient d'être purifiée. Elle aurait un PM de 31800 et un pH optimum de 8 (Tornqvist et Belfrage, 1976).

L'étape limitative dans l'hydrolyse des triglycérides du tissu adipeux semble être l'attaque de la première liaison ester car on ne voit pas s'accumuler, sauf conditions spéciales, de di- ou de monoglycérides pendant les mesures d'activité.

Nous ne nous intéresserons ici qu'aux 2 premières de ces enzymes donf le rôle physiologique est de loin le plus important : captation des acides gras circulant pour la LPL, ce qui lui confère un rôle opposé à celui de l'autre enzyme, la LHS, qui est chargée, elle, d'opérer l'hydrolyse des triglycérides contenus dans le tissu adipeux qui, on le sait, sont en effet principalement constitués d'acides gras à longues chaînes.

La lipoprotéine lipase hydrolyse les triglycérides des lipoprotéines, mais pas, comme la lipase pancréatique, les triglycérides libres. Elle s'attaque en effet aux triglycérides qui circulent dans le sang sous forme d'agrégats de haut PM liés à des protéines, des phospholipides, du cholestérol et des esters du cholestérol.

Les lipoprotéines du sang peuvent être divisées en 4 groupes (Robinson, 1970 ; Fredickson et al., 1972) en fonction de leur densité croissante : les plus légères, les chylomicrons, apparaissent dans le sang après un repas gras ; viennent ensuite les lipoprotéines de très faible densité qui proviennent principalement du foie ; finalement on décrif les lipoprotéines de faible ou de haute densité qui ne renferment que 10 p. 100 de triglycérides. Les lipoprotéines de ces 2 derniers groupes ne sont pas hydrolysées par la LPL.

Trois critères caractérisent l'activité de la lipoprotéine lipase (Korn, 1955) : activation par l'héparine, activation par un facteur protéinique du sérum qui s'identifie aux protéines des lipoprotéines, inhibition par le $\mathrm{NaCl}$ et la protamine.

Plusieurs lipoprotéines lipases sont responsables de la clarification du sang : la lipoprotéine lipase hépatique est de loin la plus active (Assmann et al., 1973) mais elle ne répond qu'au premier des critères énumérés ci-dessus, c'est-d̀-dire l'activation par l'héparine.

Le rôle de la lipoprotéine lipase du tissu adipeux a été précisé. II est admis que les triglycérides des chylomicrons sont hydrolysés non pas dans les adipocytes, mais dans les cellules endothéliales des capillaires. En effet, primitivement synthétisée dans 
les adipocyłes, la LPL migrerait dans la paroi des capillaires d'où elle exercerait son action (Scow ef al., 1972). Elle a été partiellement purifiée (environ 80 p. 100) à partir de tissu adipeux de rat (Schotz et Garfinkel, 1972). L'activité de la LPL de tissu adipeux est fortement diminuée par le jeûne et augmente en revanche chez l'animal nourri. Elle peut augmenter également par administration de glucose, d'acide nicotinique, d'actinomycine ou d'insuline. Deux fractions de lipoprotéine lipase ont été séparées à partir de tissu adipeux par chromatographie sur gel ; elles se comportent différemment. La fraction a serait principalement à l'extérieur de l'adipocyte ef représenterait la forme activée de l'enzyme, alors que la fraction b correspondrait à l'enzyme qui vient d'être synthétisée à l'intérieur de la cellule. Les deux fractions seraient stimulées par la réalimentation. En revanche, selon Garfinkel et al. (1976), l'insuline agirait de 2 façons différentes sur l'activité de la LPL. L'effet à court terme pourrait s'expliquer par une facilitation par cette hormone de la diffusion de la LPL de l'adipocyte vers les cellules de l'endothélium des capillaires où elle exerce son action. Son action à long terme, elle, résulteraif d'une stimulation de la synthèse de l'enzyme.

La lipase hormono-sensible est l'enzyme chargée d'hydrolyser les triglycérides contenus dans le tissu adipeux. Elle est inhibée par le FNa. L'addition d'albumine au milieu d'incubation est indispensable lorsque l'on veut mesurer son activité, sinon les acides gras libérés inhibent la réaction par un mécanisme de « feedback ». Une préincubation du tissu anférieure à la mesure est également favorable à la détermination de l'activité basale de l'enzyme, car elle favorise la dégradation de la noradrénaline ou de l'AMP ${ }_{\mathrm{c}}$ endogène (Appleman ef Sevilla, 1970). Elle a été purifiée 100 fois à partir de surnageant de tissu adipeux de rat par Huttunen ef al. (1970). Son PM serait voisin de 7,2.106. Elle contient environ 50 p. 100 de phospholipides. Sa purification n'a pas été conduite jusqu'à son terme et il est probable que l'on aboutira à une enzyme de PM plus faible. On ne connaît que peu de chose de sa structure, on sait seulement que les groupes $\mathrm{SH}$ paraissent indispensables à son activation par le db $\mathrm{AMP}_{\mathrm{c}}$ (Marsh et George, 1969). Sa localisation intracellulaire est discutée. Pour cerfains, elle serait sur la surface interne de la membrane interne (Crum et Calvert, 1971), pour d'autres dans la partie soluble de la cellule (Khoo et al., 1972), pour d'autres encore dans les microsomes (Wolf et al., 1975).

Les taux d'activation de la LHS en réponse aux différentes hormones lipolytiques, varient fortement d'espèce à espèce (Rudman et Di Girolamo, 1967). Selon Steinberg (1972), la lipase subirait un processus d'activation analogue à celui produit par l'AMP $_{c}$ sur la glycogène phosphorylase. Ainsi s'expliquerait le mode d'action des hormones lipolytiques sur cette lipase. Quoique se fixant sur des récepteurs différents, ces hormones semblent agir toutes sur la même adénylcyclase car leurs effets ne sont pas additifs (Butcher et al, 1968). Le schéma d'action de ces hormones invoque un phénomène en cascade, avec successivement activation de l'adényl-cyclase, augmentation de la concentration intra-cellulaire en $A M P_{c}$, activation de la kinase. L'AMP, en effet, se fixe sur la sous-unité régulatrice de cette kinase et entraîne ainsi son détachement de l'unité catalytique qui peut alors phosphoryler la lipase, la rendant ainsi active (Huttunen et al., 1970b; Walsh ef al., 1971). La mise en évidence d'une phosphatase capable d'inactiver la lipase dans des fractions non purifiées de tissu adipeux appuie cette théorie (Steinberg, 1972). 
Cependant, ce modèle ne rend pas compte de toutes les observations relatives à l'action des hormones lipolytiques sur la lipolyse. Par exemple : la concentration en $\mathrm{AMP}_{\mathrm{c}} \mathrm{n}$ 'évolue pas toujours parallèlement à la lipolyse ; le $\mathrm{GMP}_{\mathrm{c}}$ augmente la concentration d'AMP ${ }_{c}$ dans les cellules adipeuses quoiqu'il inhibe la lipolyse.

Néanmoins, on sait que les hormones n'exercent pas toutes leurs actions intracellulaires au niveau des adipocytes par l'intermédiaire de l'adénylcyclase. Elles modifient également le transport des cations (Akgün et Rudman, 1969), notamment en contrôlant l'activité de la $\mathrm{Na}^{+} \mathrm{K}+\mathrm{ATP}$ ase d'où celle de la pompe au sodium, de même que le transport des acides aminés et du glucose. Or, ces divers phénomènes sont intimement liés et toute modification survenant sur un des constituants de la membrane retentit sur un des constituants voisins.

Différences de réponse de la lipase aux principales hormones lipolytiques.

Glucagon. La méthode utilisée pour estimer la lipolyse est particulièrement critique dans les essais avec le glucagon et la mesure du glycérol libéré est nettement préférable au dosage des FFA. En effet, l'accroissement de la lipolyse induite par le glucagon s'accompagne d'une augmentation de la réestérification des acides gras libérés, grâce à la stimulation de la captation du glucose qui accompagne cette lipolyse (Lefebvre, 1972). L'âge et la taille des cellules adipeuses peuvent modifier la sensibilité des cellules au glucagon. Chez le rat, les grosses cellules semblent plus résistantes à son action, peut-être parce que la $\mathrm{P}$ diestérase y est plus importante que dans les petites (Forn et al., 1970), facilitant ainsi la dégradation de l'AMP néoformé sous l'action du glucagon. L'action lipolytique du glucagon est inhibée par l'insuline dans la plupart des espèces, mais pas chez les oiseaux, probablement parce qu'ils représentent l'espèce la plus sensible à cette hormone. On peut donc se demander, puisque ces deux hormones antagonistes sont toujours en présence, quelle est celle qui l'emporte dans des conditions physiologiques. Il est connu que pour le tissu adipeux de rat l'action antilipolytique de l'insuline est 5 fois plus forte que l'action lipolytique du glucagon, molécule pour molécule. Or, chez le rat, dans l'état post-absorptif, le rapport molaire insuline/glucagon dans le sang périphérique, est de l'ordre de 6 à 8 . Il s'abaisse à 1,5-2 après 2 à 3 jours de jeône. Puisque pour que l'effet lipolytique du glucagon se manifeste ce rapport doit passer à une valeur inférieure à 0,2 , l'effet lipolytique du glucagon ne devrait pas se produire chez le rat nourri (Lefebvre, 1975). A jeun et dans le diabète, en revanche, son rôle est plus vraisemblable, surtout si l'on considère qu'à l'effet du glucagon se surajoute celui des autres hormones lipolytiques, alors que l'insuline est à peu près seule à contrebalancer l'action de ces hormones. Les prostaglandines peuvent cependant aider l'insuline dans cette fonction puisqu'elles diminuent la concentration en $\mathrm{AMP}_{\mathrm{c}}$ dans le tissu adipeux (Butcher et al., 1958).

Catécholamines. II existe de grandes divergences dans la littérature à propos de l'action des catécholamines sur la lipolyse ef sur l'augmentation de la concentration en $\mathrm{AMP}_{\mathrm{c}}$ dans les adipocytes. Certains auteurs n'observent que peu ou pas d'augmentation de l'AMP dans ces cellules, à moins que des méthylxanthines ne leur soient associées. L'effet stimulateur des méthylxanthines sur les catécholamines est facile à expliquer puisqu'il a été montré que ces hormones stimulent l'adénylcyclase et que 
les méthylxanthines inhibent la phosphodiestérase. Elles empêchent donc l'AMP néosynthétisé sous l'action des catécholamines d'être dégradé (Vaughan et Steinberg, 1963). C'est par l'intermédiaire des récepteurs $\beta$ adrénergiques que les catécholamines stimulent l'adénylcyclase. L'existence d'un $\alpha$ récepteur mise en évidence sur les membranes des adipocytes permet plus facilement que pour d'autres hormones d'expliquer certains de leurs effets apparemment paradoxaux sur la lipolyse. Ainsi l'adrénaline diminue la concentration en $\mathrm{AMP}_{\mathrm{c}}$ dans le tissu adipeux blanc quand son effet stimulateur est bloqué par le propanolol (Turtle et Kipnis, 1967). Les nombreuses différences dans les réponses obtenues sur la lipolyse après incubation des tissus en présence de catécholamines s'expliquent facilement par la présence en plus ou moins grande quantité de tel ou tel type de récepteur et le rat n'est peut-être pas l'animal idéal pour cette étude en raison de la prédominance par rapport aux autres espèces des récepteurs $\beta$ dans son tissu adipeux (Himms-Hagen, 1970) : le lapin, p. ex., contrairement au rat, possède peu ou pas de récepteur $\beta$.

ACTH. Le lapin, comme le rat, est très sensible à l'ACTH. Comme les autres hormones lipolytiques, elle agit par stimulation de l'adénylcyclase. Nous ne signalerons ici que sa particularité de nécessiter du $\mathrm{Ca}^{+2}$ pour activer l'adénylcyclase contrairement à la plupart des autres hormones. Le Ca+2 n'est pas utile comme on l'a pensé auparavant à sa fixation sur son récepteur, il l'inhiberait plutôt et son rôle est encore mal connu (Bär et Hechter, 1969).

Thyroxine. Elle augmente in vivo et in vitrol'action des catécholamines, de l'ACTH ef du glucagon sur la lipolyse, c'est-à-dire leur effet activateur sur l'adénylcyclase (Vaughan et Steinberg, 1963). Elle n'active pas la lipolyse induite par les glucocorticoïdes ef l'hormone de croissance puisque leur mode d'action est différent de celui des autres hormones lipolytiques.

\section{Glucocorticoïdes et hormone de croissance.}

L'action lipolytique de l'hormone de croissance et des glucocorticoïdes est moins immédiate que celle des autres hormones puisqu'elle peut être bloquée par les inhibiteurs de la synthèse du DNA; elle invoque vraisemblablement la synthèse de nouvelles molécules d'adénylcyclase (Fain et al., 1971). Toutefois, le facteur sérique qui induit l'action périphérique de l'hormone de croissance sur la sulfatation du cartilage possède in vitro des actions « insulin-like»: On sait que ce facteur, encore appelé somatomédine, entre en compétition avec l'insuline pour son récepteur sur la cellule adipeuse et a ainsi, chez le rat, les mêmes actions que l'insuline.

Insuline : S'il est admis que l'effet antilipolytique de l'insuline s'accompagne d'une diminution de l'AMP , le mécanisme par lequel elle conduit à cette diminution n'est pas clair. II est connu depuis longtemps que l'insuline inhibe la lipolyse même en l'absence de glucose dans le milieu d'incubation, ce qui exclut l'intervention d'une réestérification par le glycérophosphate. On sait aussi que cette action est encore plus nette si elle est ajoutée à un tissu dont l'activité lipasique a été stimulée par de faibles quantités d'hormones lipolytiques (Jungas and Ball, 1963 ;Mahler ef al., 1964 ; Fain ef al. (1966). Quoique l'on suppose qu'elle diminue l'activité de la lipase, certains faits sont paradoxaux : 
Des concentrations d'insuline plus grandes que $100 \mu \mathrm{U} / \mathrm{ml}(0,7 \mathrm{nM})$ sont moins efficaces pour bloquer la lipolyse que des quantités plus petites (Jungas et Ball, 1963 ; Allen et Clark, 1971). L'effet antilipolytique n'est pas visible dans toutes les espèces. Les oiseaux surtout, mais aussi les lapins et la vache sont peu sensibles à cette hormone (Hagen, 1963 ; Goodridge et Ball, 1965).

Dans certaines conditions, l'insuline peut même induire une stimulation de la lipolyse plutôt qu'une inhibition. Ceci se produit quand de l'adrénaline et du glucose sont également présents dans le milieu d'incubation (Jungas et Ball, 1963 ; Hall et Ball, 1970).

La baisse de la concentration en $\mathrm{AMP}_{\mathrm{c}}$ constatée chez les animaux traités à l'insuline ne peut suffire à elle seule à expliquer l'action antilipolytique de cette hormone, car la concentration en $\mathrm{AMP}_{\mathrm{c}}$ qui serait de $5 \mu \mathrm{M}$ si elle était distribuée uniformément à travers l'eau intracellulaire (Park et al., 1969), correspond à environ 100 fois la $\mathrm{Km}$ de la protéine kinase du tissu adipeux, donc l'AMP $\mathrm{C}_{\mathrm{C}}$ est toujours présent en gros excès (Corbin ef Krebs, 1969). Ceci sous-entend que cette régulation ne peut s'effectuer que si l'AMP $P_{c}$ 'est pas uniformément distribué dans la cellule. Cette baisse de la concentration en $\mathrm{AMP}_{\mathrm{c}}$ pourrait résulter soit d'une action inhibitrice de l'insuline sur l'adénylcyclase, soit d'une action activatrice sur la phosphodiestérase qui est chargée de dégrader I'AMP.

L'effet sur l'adénylcyclase est soumis à controverse, car il semble plus visible avec de faibles concentrations en insuline qu'avec de plus grandes et n'a jamais pu être mis en évidence en présence de membranes purifiées (llliano el Cuatrecasas, 1972 ; Hepp, 1972). Il a été également proposé que l'insuline stimulait la $P$ diestérase. On sait depuis peu qu'il n'existe pas qu'une seule $P$ diestérase, mais deux: la 1 re cytoplasmique, la plus anciennement connue, a peu d'affinité pour l'AMP $P_{c}$; la $2^{\mathrm{e}}$ est liée aux membranes. L'insuline stimulerait uniquement ceffe $P$ diestérase de basse $\mathrm{Km}$ (Loten et Sneyd, 1970 ; Vaughan, 1972). Toutefois, cette action n'a pu être démontrée que lorsque l'insuline était administrée in vivo et à des rats diabétiques. Cependant, comme l'activité de la $P$ diestérase est beaucoup plus forte que celle de l'adénylcyclase, une augmentation même petite de la $P$ diestérase devrait pouvoir neutraliser l'action de l'adénylcyclase.

Il est peu probable que l'action de l'insuline résulte d'une inhibition de la protéine kinase qui est responsable de la phosphorylation de la lipase, donc de son activation ou d'une activation de son facteur inhibiteur (Ashby et Walsh, 1972), car elle ne modifie pas la lipolyse induite par le db $\mathrm{AMP}_{\mathrm{c}}$ (Fain et al., 1966). Son action semble donc résulter uniquement d'une diminution de la concentration en $\mathrm{AMP}_{\mathrm{c}}$.

Rien n'exclut non plus que l'insuline ne modifie la concentration d'un autre médiateur hormonal non encore identifié, ou encore n'agisse par l'intermédiaire du $G M P_{c}$. En effet, la concentration du $G_{M P}$ augmente dans la cellule adipeuse sous l'action de l'insuline (Illiano et al., 1973). Néanmoins, les tentatives effectuées pour détecter un effet éventuel de l'insuline sur la guanylcyclase du surnageant de foie de rat se sont révélées infructueuses (Thompson ef al., 1973). Chez les oiseaux, l'effet lipolytique du glucagon peut être supprimé par les prostaglandines ; l'insuline pourrait éventuellement agir, dans les autres espèces, en activant la phospholipase membranaire qui libère des membranes les acides gras polydésaturés précurseurs des prostaglandines. Ces dernières n'interfèrent pas non plus avec l'action du dbAMP. 
Devant un problème aussi vaste ef complexe que celui de la lipolyse les conclusions qui peuvent être tirées sont très schématiques et fragmentaires.

La fixation des hormones sur leurs récepleurs membranaires avec activation subséquente de l'adénylcyclase, d'où la concentration en $\mathrm{AMP}_{\mathrm{c}}$ intracellulaire, est un moyen commode qui permet d'expliquer la majeure partie de leurs effets activateurs sur la lipase, leur action sur l'adénylcyclase étant en effet leur action principale. On peut être étonné des différences observées chez tel ou tel animal en réponse à telle ou telle hormone, p. ex. la grande sensibilité du tissu adipeux des oiseaux au glucagon, sa faible sensibilité en revanche à l'insuline, etc. Puisqu'il est admis que ces hormones, à l'exception des glucocorticoïdes, ne franchissent pas la membrane cellulaire, la présence en plus ou moins grand nombre des divers récepteurs sur les membranes de leur tissu adipeux paraît l'explication la plus satisfaisante pour rendre compte de ces différences. Mais nous l'avons vu, les variations de la lipolyse ne suivent pas toujours les modifications de concentration en $\mathrm{AMP}_{\mathrm{c}}$. Ne tenir comple que des variations des activités de l'adénylcyclase ou de la $P$ diestérase pour expliquer la lipolyse, correspond donc à une sursimplification. Ainsi une protéine activatrice de la $P$ diestérase a été trouvée dans le fissu adipeux. La kinase elle-même permettant de phosphoryler la lipase peut également être soumise à des régulations soit dans sa synthèse, soit dans son activité.

Quoique Denton et al. (1966) aient trouvé que le contenu total en ATP du tissu adipeux ne subissait pratiquement pas de variations, que l'animal soit nourri, à jeun ou diabétique, les adipocyłes isolés peuvent présenter des fluctuations considérables des concentrations en ATP. Les modifications de la concentration en ATP ne pourraient influencer que l'activité de la lipase kinase puisque la $\mathrm{Km}$ de l'adénylcyclase pour l'AMP $_{c}$ est assez faible pour que cette enzyme ait toujours assez d'ATP à disposition (Angel ef al., 1971).

Le rôle des ions n'est sûrement pas non plus à sous-estimer puisque l'on sait que les différentes hormones lipolytiques ou l'insuline modifient leur pénétration. Cependant, comparativement à d'autres tissus le rôle du Ca semble minime dans le tissu adipeux (Krebs et al., 1968).

L'effet antilipolytique de l'insuline est, lui, beaucoup plus difficile à expliquer. S'il n'y a aucun doute qu'il s'accompagne d'une diminution de la concentration intracellulaire en $\mathrm{AMP}_{\mathrm{c}}$, on ne peut affirmer avec certitude que cette action découle plutôt d'une inhibition de l'adénylcyclase que d'une activation de la phosphodiestérase. II est possible que beaucoup de résultats négatifs concernant l'action de l'insuline sur la $P$ diestérase soient dus au fait que la présence de la $P$ diestérase membranaire est de découverte récente. Or, c'est sur celle-ci seulement que l'insuline peut agir puisque cette hormone ne franchit pas la membrane cellulaire.

\section{Régulation de la lipogenèse.}

Pour beaucoup d'espèces animales le glucose constitue le précurseur par excellence des acides gras,. Or, le tissu adipeux présente le désavantage sur le foie de ne pas être librement perméable au glucose qui dépend de l'insuline pour y pénétrer. C'est donc l'étape limitative de l'utilisation du glucose puisque, aussitôt dans la cellule, 
il est pris en charge par une hexokinase qui, contrairement à la glucokinase hépatique, possède une grande affinité pour le glucose.

L'hexokinase. L'hexokinase du tissu adipeux est adaptative in vivo : son activité diminue à jeun et augmente quand l'animal est renourri (Moore ef al, 1964). En revanche, elle diminue chez le rat diabétique alloxané, mais pas chez l'homme atteint de diabète (Galton et Wilson, 1971). Le tissu adipeux contient les 3 types d'hexokinase, toutes 3 de faible $\mathrm{Km}$. Suivant l'âge du rat, l'un des types prédomine. Le type 2 est plus abondant chez le jeune, le type 1 chez le plus âgé. L'enzyme de type 2 est la seule à être affectée par le jeûne et est plus sensible à l'insuline que celle du type 1 (Katzen, 1967). L'effet de l'insuline résulte d'un accroissement de synthèse de cette enzyme. Elle augmenterait aussi son adsorption sur les mitochondries, ce qui aurait pour effet d'augmenter la stabilité de l'enzyme (Borrebaek, 1969).

Si l'hexose $P$ isomérase ne joue qu'un rôle accessoire dans la régulation de la lipogenèse, en revanche la $P$ fructokinase $a$ une fonction importante dans l'utilisation du glucose, puisque son inhibition peut conduire à l'accumulation de glucose $6 \mathrm{P}$ qui est un inhibiteur de l'hexokinase.

Phosphofructokinase. La phosphofructokinase du tissu adipeux est également sensible à l'effet inhibiteur du jeûne (Orevi et al, 1972). Elle est inhibée par le citrate et I'ATP, alors qu'elle est activée par l'ADP, L'AMP, le phosphate, le sulfate et surtout l'AMP $_{\mathrm{e}}$ (Denton et Randle, 1966). Le rôle physiologique de l'AMP $\mathrm{P}_{\mathrm{c}}$ et des nucléotides en général sur la $P$ fructokinase du tissu n'est pas clair puisqu'on n'a pu observer de variations dans le taux des adénylnucléotides de ce tissu, à jeun, dans le diabète ou après administration d'insuline (Ballard, 1972 ; Denton ef al, 1966). Le rôle inhibiteur du citrate sur la $P$ fructokinase ne semble pas davantage avoir une importance physiologique, sauf si le fructose $6 \mathrm{P}$ existe en quantité suffisante pour augmenter la $\mathrm{Ki}$ de la $P$ fructokinase pour le citrate.

Aldolase. L'aldolase peut participer au contrôle de la glycolyse dans le tissu adipeux car, contrairement à l'aldolase du foie de rat, son activité est beaucoup plus faible que celle des enzymes responsables de phosphoryler le glucose ou le fructose $6 \mathrm{P}$ et, de plus, dans cet organe, elle diminue encore chez l'animal privé de nourriture (Orevi et al., 1972).

En revanche, la pyruvate kinase semble jouer un rôle plus accessoire comme agent de régulation de la glycolyse dans le tissu adipeux que dans le foie.

Pyruvate kinase. Tout comme celle du foie, elle existe sous 2 formes interconvertibles (Pogson, 1968) dont l'une est soumise à un contrôle stimulateur allostérique par le fructose 1,6 diP, mais la Ka de la pyruvate kinase pour le fructose 1,6 diP est plus élevée que pour l'enzyme correspondante du foie, ce qui pourrait limiter son intervention physiologique. Cependant, la concentration en fructose 1,6 diP est proportionnellement plus élevée dans le tissu adipeux que dans le foie, probablement en raison de l'absence de fructose 1,6 diphosphatase dans ce tissu (Ballard, 1972). L'alanine exerce en revanche un fort effet inhibiteur sur la pyruvate kinase, particulièrement à jeun, ce qui devrait avoir pour effet de stimuler la glycéronéogenèse dans le tissu adipeux (Seubert et Schoner, 1971). 
Pyruvate déshydrogénase (PDH). La pyruvate déshydrogénase est d'une importance capitale. C'est la seule enzyme intramitochondriale qui est réglée par phosphorylation-déphosphorylation et sur laquelle, précisément dans le tissu adipeux, on a pu démontrer une action de l'insuline. Pendant de nombreuses années, on a pensé que l'action favorable qu'exerçait l'insuline sur le tissu adipeux était due uniquement à son action sur la pénétration du glucose. On sait maintenant qu'elle contrôle directement la conversion de pyruvate en acétyl CoA en activant la pyruvate déshydrogénase.

Il s'agit d'un complexe multienzymatique dont l'activité est contrôlée par phosphorylation-déphosphorylation d'un des constituants : la pyruvate décarboxylase (Linn ef al., 1969). La forme phosphorylée de l'enzyme est la forme inactive. La phosphorylation dépend d'une kinase spécifique et de $\mathrm{Mg}^{+2}$ à faible concentration, la déphosphorylation d'une phosphatase, dont l'activité optimale nécessite la présence de concentrations élevées de $\mathrm{Mg}^{+2}$ (environ $10 \mathrm{mM}$ ), et de $\mathrm{Ca}^{+2}$, celui-ci nécessaire pour permettre sa fixation à la transacétylase. L'activité du complexe est soumise à un second type de contrôle : l'inhibition par les produits de réaction conduisant ainsi à un accroissement des rapports acétyl CoA/CoA et NADH/NAD (Reed, 1969). C'est une des raisons de l'inhibition exercée sur l'enzyme par les acides gras à longue chaîne puisque par combustion ils augmentent ces deux rapports.

Le complexe multienzymatique ne semble pas exister sous forme entièrement active, même dans les conditions physiologiques les plus favorables comme chez l'animal nourri : $1 / 6$ seulement est sous forme active dans le foie, alors que $4 / 6$ sont présents sous cette forme dans le tissu adipeux (Wieland et al., 1972 ; Jungas, 1971). L'interconversion entre les 2 formes paraît être dominée par la régulation de la PDH kinase. Cependant, aucun contrôle de cette kinase par l'AMP $P_{c}$ 'a pu être mis en évidence (Jungas, 1971). Elle est inhibée par le pyruvate, le TPP, le $\mathrm{Ca}^{+2}$. Ainsi le pyruvate favorise sa propre oxydation (Petzelt et al, 1973).

Le rôle de la pyruvate phosphatase n'est cependant pas entièrement négligeable, quoiqu'il ait été trouvé récemment que 80 P. 100 de l'activité de la pyruvate phosphatase du tissu adipeux de rat était extra-mitochondriale et ne devrait donc pas être concarnée dans la régulation de l'activité de la PDH (Stranusbie et al, 1976. Cette enzyme nécessite pour son maximum d'activité, non seulement $\mathrm{du} \mathrm{Mg}^{+2}$, mais aussi du $\mathrm{Ca}^{+2}$. Il ne semble pas néanmoins que l'insuline exerce son action activatrice sur la $\mathrm{PDH}$ en augmentant la captation du $\mathrm{Ca}^{+2}$ par les mitochondries. D'ailleurs, à l'exception peut-être du foie, la concentration en $\mathrm{Ca}^{+2}$ de la plupart des tissus est beaucoup trop grande pour jouer un rôle dans la régulation de la PDH phosphatase (Severson et al., 1976). On suppose plutôt maintenant que l'effel activateur de l'insuline résulte de son effet antilipolytique. Nous avons vu en effet que les acides gras augmentaient à la fois le NADH, l'acétyl CoA ef l'ATP intramiłochondriaux, tous trois inhibiteurs de la PDH. Mais, de plus, ils inhibent le transport extramitochondrial du citrate, qui s'accumulerait donc dans les mitochondries (Halperin et al., 1972). Or, le citrate exerce aussi une action inhibitrice sur la PDH. De plus, l'insuline augmente non seulement l'activité de la PDH, mais aussi la quantifé totale d'enzyme active et inactive ; elle contrôle donc également soit sa synthèse, soit sa dégradation (Sica et Cuatrecasas, 1973). 
Enzyme clivant le citrate. II est classiquement admis que l'acétyl CoA ne peut diffuser sous forme d'acétyl CoA hors de la mitochondrie et qu'il emprunte le plus souvent la voie du citrate pour ce faire. Après diffusion dans le cytoplasme, il subit l'action de l'enzyme clivante régénérant ainsi l'acétyl CoA. II a été démontré aussi bien dans le foie que dans le tissu adipeux des mammifères (rat, cochon), que l'enzyme clivant le citrate est une enzyme adaptative (Kornacker et Lowenstein, 1965 ; Kornacker et Ball, 1965 ; O'Hea et Leveille, 1969). Cependant, le rôle du citrate n'est probablement pas aussi absolu que l'on a bien voulu le dire, même pour les mammifères. Des différences d'espèces semblent exister et la souris, par ex€mple, utilise moins efficacement ce transporteur que le rat. En effet, dans le tissu adipelx , le citrate $2,4-14 \mathrm{C}$ s'incorpore 20 fois plus environ que le 1,5 (Rous, 1971). Ces résultats laissent supposer que l'enzyme clivant le citrate est peu active dans le tissu adipeux des mammifères, comme l'ont confirmé des mesures directes de l'activité de l'enzyme clivant le citrate (Rous, 1974).

Nous ne reviendrons pas sur les deux mécanismes d'élongation mitochondrial et microsomal peu actifs, surtout dans le tissu adipeux, ef considérerons maintenant les enzymes du mécanisme de synthèse par condensation qui est de loin le plus important. Cette synthèse s'effectue en 2 étapes : La première est catalysée par l'acétyl CoA carboxylase qui fixe le $\mathrm{CO}_{2}$ sur l'acétyl CoA grâce à l'énergie de l'ATP, donnant ainsi du malonyl CoA ; - la deuxième, par la synthétase des acides gras : 7 molécules de malonyl CoA se condensent avec une molécule d'acétyl CoA pour donner naissance à une molécule d'acide palmitique. En réalité, cette condensation ne s'effectue pas directement et les dérivés CoA doivent d'abord se fixer au groupement $\mathrm{SH}$ d'une protéine, l'ACP, qui n'est autre que la partie centrale du complexe enzymatique que constitue la synthétase des acides gras. L'hydrogène est apporté par le NADPH.

Acéfyl CoA Carboxylase (ACX) : son coenzyme est la biotine. (Wakil ef al., 1958). Le $\mathrm{CO}_{2}$ se fixe d'abord sur le NH de la molécule de biotine et, dans un second temps, la lysine qui la relie à l'apoenzyme fonctionnant comme un bras flexible, déplace la carboxybiotine vers le site de fixation, l'acétyl CoA, lui permettant ainsi d'être carboxylé en malonyl CoA (Guchhait ef al, 1971).

L'ACX a été purifiée à partir de tissu adipeux de bœuf par Moss et al. $(1969,1972)$ et de rat par Martin et al. (1961). Elle peut exister comme celle du foie sous forme de monomères de PM d'environ 560000 ou de polymères (Moss et al., 1969). La présence de citrate favorise également dans ce tissu la polymérisation de l'enzyme, la rendant ainsi beaucoup plus active. Cette activation se traduit par une augmentation de la $V$ max de l'enzyme pour l'ATP, le bicarbonate ou l'acétyl CoA, sans modification de la $\mathrm{Km}$ (Moss et al, 1972). La réactivation par le citrate est presque instantanée dans ce tissu, contrairement à ce qui se passe avec l'enzyme du foie. L'importance physiologique du citrate est plus difficile à prouver. Les concentrations en citrate sont insuffisantes pour assurer cette stimulation, à moins que sa répartition intracyloplasmique ne soit pas uniforme (Volpe ef Vagelos, 1973b).

Le palmityl CoA exerce une inhibition compétitive vis-à-vis de l'acétyl CoA (Goodridge, 1972). Cette inhibition peut expliquer pourquoi, à jeun, la lipogenèse baisse parfois plus que ne le laisserait escompter la baisse d'activité enzymatique mesurée in vitro.

La réponse à une carence en biotine est plus nette dans le tissu adipeux que dans 
le foie (Mayobre et al., 1975 ; Jacobs et al., 1970). L'activité de l'enzyme du foie (ACX) est environ 5 fois moins inhibée. 40 p. 100 de l'ACX du foie el 90 p. 100 de celle du tissu adipeux seraient respectivement sous forme d'apoenzyme chez les animaux carencés en biotine (Landman et Dakshinamurti, 1973).

Une inhibition par l'AMP ${ }_{c}$ a également été proposée. Pour Carlson et Kim (1973), l'ACX pourrait exister sous forme phosphorylée ou non phosphorylée. la forme phosphorylée étant la forme inactive. Cette théorie n'a pas été confirmée par Lane et al. (1974). L'AMP ${ }_{c}$ inhibe la synthèse des acides gras dans le foie, mais il ne s'agit peutêrre là que d'une action indirecte résultant de l'action lipolytique de cette substance. In vivo, dans des conditions où l'AMP $\mathrm{P}_{\mathrm{c}}$ produisait une forte hyperglycémie et une stimulation de la gluconéogenèse, aucune action de l'AMP $\mathrm{P}_{\mathrm{c}}$ sur la synthèse des acides gras dans le tissu adipeux de souris n'a été constatée, alors qu'une inhibition était enregistrée dans le foie. Le $\mathrm{GMP}_{\mathrm{c}}$ stimulait légèrement cette synthèse dans les organes extrahépatiques (Bron et Rous, 1971).

Les effets des métabolites sur l'acétyl CoA carboxylase sont des effets à court terme, que les hormones elles-mêmes peuvent aussi entraîner. L'insuline, par exemple en inhibant la lipolyse stimulerait l'activité de l'enzyme (Halestrap et Denton, 1974). Cependant, tout comme la synthétase des acides gras, l'acétyl CoA carboxylase ne subit pas uniquement une régulation à court terme. Comme les réponses évoluent parallèlement pour ces deux enzymes, nous les traiterons simultanément après avoir examiné les régulations à court terme de la synthétase des acides gras.

Synthétase des acides gras (FAS). La synthétase des acides gras est le complexe enzymatique responsable de la synthèse d'acide palmitique à partir d'acétyl CoA et de malonyl CoA. Toutefois, dans le tissu adipeux, l'acétyl CoA serait avantageusement remplacé par le butyryl CoA (Lin et Kumar, 1972).

Le mécanisme de la régulation rapide de la synthétase esł mal connu. Des modifications de son activité ont été attribuées à certains métabolites. Une stimulation par les hexoses diphosphates (Wakil et al., 1958 ; Porter ef al., 1971), une inhibition par le palmityl CoA ef le malonyl CoA ont été décrites, quoique le rôle physiologique de ces facteurs ne soit pas encore établi. Volpe et Vagelos (1973a) ont également recherché si le renouvellement du groupement prosthétique de l'ACP, c'est-à-dire la 4'phosphopanthétéine contrôlait l'activité globale de la synthétase des acides gras. Son renouvellement est extrêmement rapide : 4-5 h. Cependant, quoique le jeûne diminue l'échange de la 4'phosphopanthétéine entre le CoA et l'ACP et que le régime sans graisse au contraire l'accroisse, le renouvellement du groupe prosthétique ne semble jouer aucun rôle important dans la régulation de l'activité de la FAS car les études d'immunoprécipitation ont montré que tous les points d'équivalence étaient égaux. Ceci exclut la présence de molécules de synthétase incomplètes, donc sous forme inactive, de même qu'un rôle régulateur par le palmityl CoA ou d'autres métabolites activateurs ou inhibifeurs, car ces espèces activées ou inhibées devraient être immunologiquement réactives. Les changements d'activilé de l'enzyme correspondent donc pour la synthétase des acides gras et, contrairement à l'acétyl CoA carboxylase seulement, à des changements dans la concentration en enzyme. 


\section{Régulation à long terme de l'ACX et de la FAS.}

C'est elle qui contrôle leur concentration. Elle dépend avant tout de facteurs alimentaires et des hormones.

- Facteurs alimentaires. Si le taux de synthèse el de dégradation de l'ACX dans le foie de rat est bien connu, aucune étude de ce type n'a été réalisée à ce jour dans le tissu adipeux et on aurait bien tort d'extrapoler au tissu adipeux les résultats obtenus pour le foie. Nous avons pu constater en effet qu'une simple préincubation avec le citrate suffit à lever l'inhibition exercée par le jeûne sur l'acétyl CoA carboxylase du tissu adipeux de rat, alors qu'elle était sans effet sur le foie, ce qui laisserait supposer que la concentration d'ACX est moins vite abaissée par le jeûne dans le tissu adipeux que dans le foie. Ce serait en général (diabète, obésité), plus la synthèse que la dégradation qui conditionnerait l'activité de l'enzyme (Nakanishi et Numa, 1970 ; Craig ef al., 1972). Les dégradations de ces enzymes ne seraient efficaces comme objet de régulation qu'à jeun (Majerus ef Kilburn, 1969).

- Action des hormones sur les enzymes de la synthèse des acides gras. Les quelques articles consacrés à l'étude de l'action des hormones sur la synthèse des enzymes dans le tissu adipeux de rat ont montré que, contrairement à ce qui se passait dans le foie, l'activité de la FAS et celle de l'ACX du tissu adipeux de rat étaient abaissées par administration de glucocorticoïdes (Diamant et Shafrir, 1975 ; Volpe et Marasa, 1975), alors que dans le foie c'est le glucagon qui exerce une action inhibitrice sur la synthèse de ces enzymes (Volpe et Marasa, 1975 ; Lakshmanan ef al, 1972). En revanche l'insuline stimule la synthèse de la FAS et de l'ACX aussi bien dans le foie que dans le tissu adipeux (Nakanishi et Numa, 1970 ; Lakshmanan et al., 1975); des effets divergents ont été obtenus suivant les auteurs en ce qui concerne l'action des hormones thyroïdiennes (Roncari et Murthy, 1975 ; Diamant et al., 1972).

Enzymes impliquées dans la synthèse du NADPH cytoplasmique.

Trois systèmes sont impliqués dans la formation du NADPH cytoplasmique :

1) la voie oxydative du glucose. Elle est particulièrement active dans le tissu adipeux de rat ou de porc puisqu'elle peut fournir dans ce tissu jusqu'à 60 p. 100 du NADPH nécessaire à la synthèse des acides gras (Flatt et Ball, 1964 ; Katz ef al., 1966).

Deux des enzymes de la voie des pentoses catalysent une réaction formant du NADPH. Ce sont : la glucose $6 \mathrm{P}$ déshydrogénase et la $6 \mathrm{P}$ gluconate déshydrogénase. La glucose $6 P$ déshydrogénase est très sensible au jeûne et n'importe quel type de réalimentation ne peut suffire à compenser l'effet inhibiteur produit par l'absence de nourriture. L'association de glucose, protéines ef insuline est indispensable (Szepesi ef Freedland, 1968). Contrairement à son action sur l'enzyme hépatique le fructose inhibe la synthèse de la glucose 6 PDH dans le tissu adipeux (Chevalier ef al, 1972). Les acides gras, particulièrement non saturés, ont aussi une action inhibitrice (Wiley et Leveille, 1973). L'activité de la glucose 6 PDH dépend plus de l'importance de la synthèse des acides gras qui consomment le $\mathrm{NADPH}$, que de la concentration en glu- 
cose $6 P$, puisque sa concentration excède de plus de $3 \times$ la valeur de la $\mathrm{Km}$ de l'enzyme (Greenbaum ef al., 1971). Il a été proposé que l'AMP $\mathrm{c}_{\mathrm{c}}$ inhibe la synthèse de cette enzyme dans le foie ; aucune preuve de cela n'a été fournie pour le tissu adipeux.

Comparativement au porc, la voie oxydative est peu active chez les ruminants (Baldwin et al., 1973 ; Ingle ef al., 1972) qui d'ailleurs ne reçoivent que peu de glucose de leur alimentation. Les 2 autres sources de NADPH sont donc plus opératives dans ces espèces.

2) La voie de l'isocitricodéshydrogénase fournit dans le cyłoplasme une molécule de NADPH lors de l'oxydation de l'isocitrate en acide oxalosuccinique.

3) Cette voie est assez peu efficace chez le rat qui lui préfère la voie de l'enzyme malique : la réaction catalysée par cette enzyme est très importante car elle permet au malate de fournir du NADPH lors de sa décarboxylation en acide pyruvique et de récupérer ainsi une partie du NADH issu de la glycolyse après l'avoir préalablement fixé sur l'acide oxaloacétique provenant du clivage du citrate (Allee ef al. (1972) ; Wise et Ball, 1964. L'enzyme malique du tissu adipeux est adaptative alors que dans le foie deux isoenzymes existent dont un seul est adaptatif (Saito et Tomita, 1973).

\section{Acétyl CoA synthétase :}

Le glucose n'est pas le seul substrat capable de fournir l'acétyl CoA. L'acétate représente pour les ruminants le meilleur précurseur de la synthèse des acides gras (Annison et al, 1957 ; Reid et al, 1957). Chez les herbivores, en effet, il peut se former en abondance grâce aux fermentations intestinales. Cependant, même chez les mammifères, dans le tissu adipeux en particulier, le citrate n'est pas la source exclusive d'acétyl CoA et il est connu que l'acétate (Barth et al., 1972) et l'acétoacétate (Rous et Favarger, 1973 ; Rous, 1976a) peuvent aussi, suivant les espèces animales, être de meilleurs précurseurs des acides gras que le glucose. Chez l'homme, l'importance de l'acétate pourrait aussi être non négligeable puisqu'il constitue le produit d'oxydation de l'alcool éthylique (Krebs et Perkins, 1970).

La diffusion de l'acétyl CoA sous forme d'acétyl carnitine hors des mitochondries paraît peu importante (Greville, 1969).

L'acétate une fois parvenu dans le cytoplasme de la cellule doit y être activé en acétyl CoA afin de pouvoir participer à la synthèse des acides gras. L'enzyme correspondante, l'acétyl CoA synthétase, doit donc jover un rôle capital dans la régulation de la synthèse des acides gras de plusieurs espèces animales, en particulier chez les animaux dont l'activité de l'enzyme clivant le citrate est très faible. L'acétyl CoA synthétase cytoplasmique est d̀ nouveau une enzyme adaptative (Barth ef al., 1972).

II est intéressant de noter que dans le tissu adipeux où l'utilisation de l'acétate pour des synthèses semble particulièrement utile, le rapport des activités de l'acétyl CoA synthétase/acétyl CoA hydrolase est en faveur de l'acétyl CoA synthétase alors que c'est le contraire qui se produit dans le foie. Dans le même ordre d'idée, on constate que l'acétyl CoA synthétase cytoplasmique de tissu adipeux de rat est davantage stimulée par la réalimentation que celle du foie (Barth et al., 1972). De plus, tout comme l'ACX elle est inhibée par les acyl CoA à longue chaîne. 
Acéfoacétyl CoA synthétase :

Il a été démontré que l'acétoacétate s'incorporait dans les acides gras du tissu adipeux humain beaucoup mieux que le glucose qui est un mauvais précurseur (Kissebah et al., 1974). L'acétoacétate n'est pas seulement un bon précurseur des acides gras mais peut être considéré également comme un bon transporteur d'acétyl CoA puisqu'il a été démontré récemment que la synthèse des corps cétoniques n'était pas seulement l'apanage du foie mais que les mitochondries du tissu adipeux possédaient également des enzymes capables de synthétiser l'acétoacétate libre (Rous, 1976b). La synthèse de l'acétoacétyl CoA synthétase tout comme celle de l'acétyl CoA synthétase est contrôlée par l'insuline (Rous, 1977).

Les enzymes de la synthèse des acides gras sont soumises à 2 types de régulation : une régulation à court terme qui se traduit uniquement par des modifications d'activité des enzymes, et une régulation à long terme résultant des modificctions de la concentration de ces enzymes. Aucun contrôle ne semble s'exercer par l'intermédiaire des systèmes enzymatiques qui fournissent le NADPH qui paraît être toujours en excès dans la cellule, de même que l'acétyl CoA ou encore l'ATP (Veech et Guynn, 1974). En revanche, la régulation est sous la dépendance de la concentration en malonyl CoA, de l'activité de l'acétyl CoA carboxylase et de la synthèse à la fois de l'ACX et de la FAS.

La régulation à long terme de la synthèse des acides gras implique des modifications survenant aussi bien sur la FAS que sur l'ACX. L'insuline, par exemple, augmente la synthèse des 2 enzymes, alors qu'au contraire les glucocorticoïdes l'inhibent. II est probable que le mécanisme par lequel ces deux hormones modifient la synthèse de ces enzymes découle de leur action sur la pénétration du glucose dans le tissu adipeux ; l'insuline augmentant cette pénétration induit la synthèse de nouvelles molécules d'enzymes ; les glucocorticoïdes qui, au contraire, diminuent la captation du glucose, agissent comme répresseurs. Il semble que plus que le glucose, ce soit un métabolite de ce composé qui intervient dans cette régulation, ef que son action résulte de l'augmentation du taux du $\mathrm{GMP}_{\mathrm{c}}$. En ce qui concerne la régulation à court terme, seule l'ACX paraît intervenir. On a longtemps considéré que l'ACX était l'enzyme limitative de la synthèse des acides gras, car son activité mesurée in vitro était inférieure à celle de la synthétase des acides gras. Cependant il n'est pas exclu que les différences primitivement constatées soient dues uniquement à l'utilisation, pendant les mesures, d'un pH plus favorable à l'activité de la FAS qu'a celle de l'ACX (Chang et al., 1967).

Le contrôle s'exerçant sur l'ACX à court terme dépend avant tout de la concentration en acides gras à longues chaînes. Ceux-ci agissent comme inhibiteurs compétitifs de l'acétyl CoA. En outre, en s'opposant à la diffusion du citrate hors de la mitochondrie, ils provoquent chez les animaux qui utilisent ce substrat pour transporter l'acétyl CoA hors des mitochondries, un déficit en acétyl CoA cytoplasmique; de plus, le citrate lui-même, nous l'avons vu, peut accroître encore cetle carence en inhibant la pyruvate déshydrogénase.

Les deux phénomènes que nous avons étudiés, lipolyse ef lipogenèse, sont donc étroitement liés. Les hormones lipolytiques en augmentant la concentration intracellulaire en acides gras entraînent une inhibition de l'ACX. Inversement, l'insuline 
s'oppose doublement à l'action défavorable qu'exercent les acides gras sur la lipogenèse ; d'une part, en augmentant la captation du glucose elle accroît les stocks en glycéro-P indispensables à la réestérification des acides gras, d'autre part, en diminuant la concentration en $\mathrm{AMP}_{\mathrm{c}}$ elle inactive la lipase d'où diminue la lipolyse. C'est l'hormone lipogénique par excellence puisqu'elle contrôle également la régulation à long terme dans le tissu adipeux en induisant la synthèse de l'ACX et de la FAS.

\section{Réunion Groupe Développement INRA/Productions animales Thiverval-Grignon, 14-15 avril 1976.}

\section{Références}

AKGÜN S., RUDMAN D., 1969. Relationships between mobilization of free fatty acids from adipose tissue, and the concentration of calcium in the extracellular fluid and in the tissue. Endocrinology, 84, 926-930.

ALLEE G. L., ROMSOS D. R., LEVEILLE G. A., BAKER D. A., 1972. Metabolic adaptation induced by meal-eating in the pig. J. Nutr., 102, 1115-1122.

ALLEN D. O., CLARK J. G., 1971. Effect of various antilipolytic compounds on adenyl cyclase and phosphodiesterase activity in isolated fat cells. Adv. enzym. Reg., 9, 99.

ANGEL A., DESAI K., HALPERIN M. L., 1971. Free fatty acid and ATP levels in adipocytes during lipolysis. Metabolism, 20, 87-99.

ANNISON E. F., HILL K. J., LEWIS D., 1957. Studies on the portal blood of sheep. Absorption of volatile fatty acid from the rumen of the sheep. Biochem. J., 66, 592-599.

APPLEMAN M. M., SEVILLA C. L., 1970. In GREENGARD P., COSTA E. Role of cyclic AMP in cell function, Adv. Biochem. Psychopharmacol., 3, 209.

ASHBY C. D., WALSH D. A., 1972. Assessement of the role of a protein inhibitor of cyclic AMP dependent protein kinases. Fed. Proc., 31, 1250.

ASSMANN G., KRAUSS R. M., FREDICKSON D. S., LEVY R. I., 1973. Characterization subcellular localization, and partial purification of a heparin released triglyceride lipase from rat liver. J. biol. Chem., 248, 1992-1999.

BALDWIN R. L., REICHL J. R., LOUIS S., SMITH N. E., YANG Y. T., OSBORNE E., 1973. Effects of age pregnancy and lactation on rat, guinea pig and cow adipose enzyme activities and cow adipose metabolism. J. Dairy Sci., 56, 340-349.

BALLARD F. J., 1972. Effects of fasting and refeeding on the concentrations of glycolytic intermediates and the regulation of lipogenesis in rat adipose tissue in vivo. Biochim. biophys. Acta, 273, 110-118.

BAR H. P., HECHTER O., 1969. Adenyl cyclase and hormone action. III. Calcium requirement for ACTH stimulation of adenyl cyclase. Biochem. biophys. Res. Commun., 35, 681-686.

BARTH C., SLADEK M., DECKER K., 1972. Dietary changes of cytoplasmic acetyl-CoA synthetase in different rat tissues. Biochim. biophys. Acta, 260, 1-9.

BORREBAEK B., 1969. Mitochondrial-bound hexokinase of the rat epididymal adipose tissue and its possible relation to the action of insulin. Biochem. Med., 3, 485-497.

BOYER J., 1975. Sur quelques aspects actuels de l'activité lipolytique du tissu adipeux humain. $C$. $R$. Soc. Biol., 169, 1404-1412.

BRON T., ROUS S., 1971. Comparaison de quelques effets du 3', 5' GMP cyclique et du 3', 5' AMP cyclique sur le métabolisme glucolipidique de la souris vivante. Biochim. biophys. Acta, 237, 156-166.

BUTCHER R. W., BAIRO C. E., SUTHERLAND E. W., 1968 . Effect of lipolytic and antilipolytic substances on adenosine $3^{\prime}, 5^{\prime}$ monophosphate levels in isolated fat cells. J. biol. Chem., 243, $1705-1712$.

CARLSON C. A., KIM K. H., 1973. Regulation of hepatic acetyl coenzyme A carboxylase by phos phorylation and dephosphorylation. J. biol. Chem., 248, 378-380.

CHANG H., SEIDMAN I., TEEBOR G., LANE M. D., 1967. Liver acetyl CoA carboxylase and fatty acid synthetase relative activities in the normal state and in hereditary obesiły. Biochem. biophys. Res. Commun., 28, 682-686. 
CHEVALIER M. M., WILEY J. H., LEVEILLE G. A., 1972. Effect of dietary fruciose on fatty acid synthesis in adipose tissue and liver of the rat. J. Nutr., 102, 337-342.

CORBIN J. D., KREBS E. G., 1969. A cyclic AMP. Stimulated protein kinase in adipose tissue. Biochem., biophys. Res. Commun., 36, 328-336.

CRAIG M. C., NEPOKROEFF C. M., LAKSHMANAN M. R., PORTER J. W., 1972 Effect of dietary change on the rates of synthesis and degradation of rat liver fatty acid synthetase. Arch. Biochem. Biophys., 152, 619-630.

CRUM L. R., LECH J., 1969. Characteristics and distribution of fluoride-sensitive tributyrinase in rat tissues. Biochim. biophys. Acta, 178, 508-518.

CRUM L. R., CALVERT D. N., 1971. Lipolytic activity of adipocyle ghosts. Biochim. biophys. Acta, 225, $161-165$.

DENTON R. M., RANDLE P. J., 1966. Citrate and the regulation of adipose tissue phosphofructokinase. Biochem. J., 100, 420-423.

DENTON R. M., YORKE R. E., RANDLE P. J., 1966. Measurement of concentrations of metabolites in adipose tissue and effects of insulin, alloxan-diabetes and adrenalin. Biochem. J., 100, 407-419.

DIAMANT S., SHAFRIR E., 1975. Modulation of the activity of insulin-dependent enzymes of lipogenesis by glucorticoids. Eur. J. Biochem., 53, 541-546.

DIAMANT S., GORIN E., SHAFRIR E., 1972. Enzyme activities related to fatty acid synthesis in liver and adipose tissue of rats treated with triiodothyronine. Eur. J. Biochem., 26, 553-559.

FAIN J. N., DODD A., NOVAK L., 1971. Relationship of protein synthesis and cyclic AMP to lipolytic action of growth hormone and glucocorticoids. Metabolism, 20, 109-118.

FAIN J. N., KOVACEC V. P., SCOW R. O., 1966. Antilipolytic effect of insulin in isolated fat cells of the rat. Endocrinology, 78, 773-778.

FLATT J. P., BALL E. G., 1964. Studies on the metabolism of adipose tissue. XV. An evaluation of the major pathways of glucose catabolism as influenced by insulin and epinephrine. J. biol. Chem., 239, 675-685.

FORN J., SCHONHOFER P. S., SKIDMORE I. F., KRISHNA G., 1970. Effect of aging on the adenyl cyclase and phosphodiesterase activity of isolated fat cells of rat. Biochim. biophys. Acta, 208, 304-309.

FREDICKSON D. S., LUX S. E., HERBERT P. N., 1972. In W. L. HOLMES, R. PAOLETTI, D. KRITCHEVSKY. Pharmacological Control of Lipid Metabolism, p. 25, Plenum, New-York.

GALTON D. J., WILSON J. P., 1971. The effect of starvation and diabetes on glycolytic enzymes in human adipose tissue. Clin. Sci., 41, 545-553.

GARFINKEL A. S., NILSSON-EHLE P., SCHOTZ M., 1976. Regulation of lipoprotein lipase. Induction by insuline. Biochim. biophys. Acta, 424, 264-273.

GOODRIDGE A. G., BALL E. G., 1965. Studies on the metabolism of adipose tissue. XVIII. In vitro effects of insuline, epinephrine and glucagon on lipolysis and glycolysis in pigeon adipose tissue. Comp. Biochem. Physiol., 16, 367-381.

GOODRIDGE A. G., 1972. Regulation of the activity of acetyl coenzyme A carboxylase by palmitoyl coenzyme A and citrate. J. biol. Chem., 247, 6946-6952.

GREENBAUM A. L., GUMAA K. A., MCLEAN P., 1971. The distribution of hepatic metabolites and the control of the pathways of carbohydrate metabolism in animals of different dietary and hormonal status. Arch. Biochem. Biophys., 143, 617-663.

GREVILLE G. D., 1969. In J. M. LOWENSTEIN «Citric acid cycle control and compartmentation » p. 1, Dekker, New York.

GUCHHAIT R. B., MOSS J., SOKOLSKIS W., LANE M. D., 1971. The carboxyl transferase component of acetyl CoA carboxylase : structural evidence for intersubunit translocation of the biotin prosthetic group. Proc. nat. Acad. Sci., USA, 68, 653-657.

HAGEN J. H., 1963. The effect of insulin on concentration of plasma glycerol. J. Lipid Res., 4, 46-51. HALESTRAP A. P., DENTON R. M., 1974. Hormonal regulation of adipose tissue acetyl CoA carboxylase by changes in the polymeric state of the enzyme. The role of long chain fatty acyl CoA thioesters and citrate. Biochem. J., 142, 365-377.

HALL G. L., BALL E. G., 1970. Factors affecting lipolysis rates in adipose tissue. Biochem. biophys. Acta, 210, 209-220.

HALPERIN M. L., ROBINSON B. H., FRITZI. B., 1972. Effects of palmitoyl CoA on citrate and malate transport by rat liver mitochondria. Proc. nat. Acad. Sci., USA, 69, 1003-1007. 
HEPP K. Y., 1972. Adenylate cyclase and insulin action. Eur. J. Biochem., 31, 266-276.

HIMMS-HAGEN J., 1970. Adrenergic receptors for metabolic responses in adipose tissue. Fed. Proc., 29, 1388-1401.

HUTTUNEN J. K., AQUINO A. A., STEINBERG D., 1970a. A purified triglyceride lipase, lipoprotein in nature, from rat adipose tissue. Biochim. biophys. Acta, 224, 295-297.

HUTTUNEN J. K., STEINBERG D., MAYER S. E., 1970b. Protein kinase activation and phosphorylation of a purified hormone sensitive lipase. Biochem. biophys. Res., Commun., 41, 1350-1356.

ILLIANO G., CUATRECASAS P., 1972. Modulation of adenylate cyclase activity in liver and fat cell membranes by insulin. Science, 175, 906-908.

ILLIANO G., TELL G. P., SIEGEL M. J. CUATRECASAS P., 1973. Guanosine 3' : 5'-cyclic monophosphate and the action of insulin and acelyl choline. Proc. nat. Acad. Sci., U.S.A., 70, 2443-2447

INGLE D. L., BAUMAN D. E., GARRIGUS U. S., 1972. Lipogenesis in the ruminant : in vitro study of tissue sites, carbon source and reducing equivalent generation for fatty acid synthesis. J. Nutr., 102, 609-616.

JACOBS R., KILBURN E., MAJERUS P. W., 1970. Acetyl coenzyme A carboxylase. The effects of biotin deficiency on enzyme in rat liver and adipose tissue. J. biol. Chem., 245, 6462-6467.

JENSEN R. G., 1971. Lipolytic enzymes. Progr. Chem. Fats and other Lipids, 11, 347-394.

JUNGAS R. L., 1971. Hormonal regulation of pyruvate dehydrogenase. Metab. clin. Exp., 20, 43-53.

JUNGAS R. L., BALL E. G., 1963. Studies on the metabolism of adipose tissue. XII. The effects of insulin and epinephrine on free fatty acid and glycerol production in the presence and absence of glucose. Biochemistry, 2, 383-388.

KATZ J., LANDAU B., BARTSH G. E., 1966. The pentose cycle, triose phosphate isomerization and lipogenesis in rat adipose tissue. J. biol. Chem., 241, 727-740.

KATZEN H. M., 1967. The multiple forms of mammalian hexokinase and their significance to the action of insulin. Adv. Enz. Regul., 5, 335.

KHOO J. C., JARETT L., MAYER S. E., STEINBERG D., 1972. Subcellular distribution of and epinephrine induced changes in hormone sensitive lipase, phosphorylase and phosphorylase kinase in rat adipocytes. J. biol. Chem., 247, 4812-4818.

KISSEBAH A. H., TULLOCH B. R., FRASER T. R., 1974. Interrelationship between glucose and acetoacetate metabolism in human adipose tissue. Diabetologia, 10, 69-75.

KORN E. D., 1955. Clearing-factor : a heparin activated lipoprotein lipase. Il. Substrate specificity and activation of coconut oil. J. biol. Chem., 215, 15-26.

KORNACKER M. S., LOWENSTEIN J. M., 1965. Citrate and the convertion of carbohydrate into fat. The activities of citrate cleavage enzyme and acetate thiokinase in livers of starved and re-fed rats. Biochem. J., 94, 209-215.

KORNACKER M. S., BALL E. G., 1965. Citrate cleavage in adipose tissue. Proc. nat. Acad. Sci., U.S.A., 54, 899-904.

KREBS E. G., HUSTON R. B., HUNKELER F. L., 1968. Properties of phosphorylase kinase and its control in skeletal muscle. Adv. Enz. Regul., 6, 275.

KREBS H. A., PERKINS J. R., 1970. The physiological role of liver alcohol dehydrogenase. Biochem. J., 118, 635-644.

LAKSHMANAN M. R., NEPOKROEFF C. M., KIM M., PORTER J. W., 1975. Adaptive synthesis of fatty acid synthetase and acetyl CoA carboxylase by isolated raf liver cells. Arch. Biochem. Biophys., 169, 737-745.

LAKSHMANAN M. R., NEPOKROEFF C. M., PORTER J. W., 1972. Control of the synthesis of fatty acid synthetase in rat liver by insulin, glucagon, and adenosine 3': 5' cyclic monophosphate. Proc. nat. Acad. Sci., USA, 69, 3516-3519.

LANDMAN A. D., DAKSHINAMURTI K., 1973. Isolation of acetyl CoA apocarboxylase by sepharose avidin affinity chromatography. Analyt. Biochem., 56, 191-195.

LANE M., MOSS J., POLAKIS S. E., 1974. Acetyl coenzyme A carboxylase. Curr. Top. Cell. Regul., 8, 139-195.

LEFEBVRE P., 1972. In P. J. LEFEBVRE, R. H. UNGER. Glucagon, molecular physiology, clinical and therapeutical implications, P. 61, Pergamon Press, Oxford.

LEFEBVRE P., 1975. Glucagon and adipose tissue. Biochem. Pharmacol., 24, 1261-1266.

LIN C. H., KUMAR S., 1972. Pathway for the synthesis of fatty acids in mammalian tissues. J. biol. Chem., 247, 604-606. 
LINN T. C., PETTIT F. H., REED L. J., 1969. $\propto$-Keto acid dehydrogenase complexes. X. Regulation of the activity of the pyruvate dehydrogenase complex from beef kidney mitochondria by phosphorylation and dephosphorylation. Proc. nat. Acad. Sci., USA, 62, 234-241.

LOTEN E. G., SNEYD J. G. T., 1970. An effect of insulin on adipose tissue adenosine 3' : 5' cyclic monophosphate phosphodiesterase. Biochem. J., 120, 187-193.

MAHLER R., STAFFORD W. S., TARRANT M. E., ASHMORE J., 1964. The effect of insulin on lipolysis. Diabetes, 13, 297-302.

MAJERUS P. W., KILBURN E., 1969. Acetyl coenzyme A carboxylase. The role of synthesis and degradation in regulation of enzyme levels in rat liver. J. biol. Chem., 244, 6254-6262.

MARSH D. G., GEORGE J. M., 1969. Importance of sulphydryl groups for lipolytic action in the isolated fat cell. J. biol. Chem., 244, 1381-1382.

MARTIN D. B., HORNING M. G., VAGELOS P. R., 1961. Fatty acid synthesis in adipose tissue. I. Purification and properties of a long chain fatty acid-synthezing system. J. biol. Chem., 236, 663-668.

MAYOBRE M. L., VEUTEY F., ROUS S., FAVARGER P., 1975. Production rapide d'une carence en biotine chez la souris. C. R. Soc. Biol., 169, 892-898.

MOORE R. O., CHANDLER A. M., TETTENHORST N., 1964. Glucose-ATP transferases in adipose tissue of fasted an refed rats. Biochem. biophys. Res. Commun., 17, 527-531.

MOSS J., YAMAGISHI M., KLEINSCHMIDT A. K., LANE M. D., 1969. Acetyl CoA carboxylase : properties of the mammalian adipose tissue enzyme. Fed. Proc., 28, 538.

MOSS 3., YAMAGASHIM., KLEINSCHMIDTA. K., LANE M. D., 1972. Acetyl coenzyme A carboxylase. Purification and properties of the bovine adipose tissue enzyme. Biochemistry, 11, 3779-3786.

NAKANISHI S., NUMA S., 1970. Purification of rat liver acetyl conzyme. A carboxylase and immunochemical studies on its synthesis and degradation. Eur. J. Biochem., 16, 161-173.

O'HEA E. K., LEVEILLE G. A., 1969. Influence of fasting and refeeding on lipogenesis and enzymatic activity of pig adipose tissue. J. Nutr., 99, 345-352.

OREVI M., GORIN E., SHAFRIR E., 1972. Adaptive changes of phosphofructokinase and aldolase in adipose tissue. Eur. J. Biochem., 30, 418-426.

PARK C. R., SNEYD J., CORBIN J. D., JEFFERSON L., EXTON J., 1969. In Proc. 6th Congr. Int. Diabetes Fed., p. 5, Excepta Medica, Amsterdam.

PETZELT C., LOFFLER G., WIELAND O. H., 1973. Inferconversion of pyruvate dehydrogenase in the isolated perfused rat liver. Eur. J. Biochem., 33, 117-122.

POGSON C. I., 1968. Two interconvertible forms of pyruvate kinase in adipose tissue. Biochem. biophys. Res. Commun., 30, 297-302.

PORTER J. W., KUMAR S., DUGAN R. E., 1971. Synthesis of fatty acids by enzymes of avian and mammalian spacies. Progr. Biochem. Pharmacol., 6, 1-101.

REED L. J., 1969. Pyruvate deshydrogenase complex. Curr. Top. Cell. Reg., 1, $233-251$.

REID R. L., HOGAN J. P., BRICKS P. K., 1957. The effect of diet on individual volatile fatty acids in the rum $n$ of sheep, with particular reference to the effect of low rumen $\mathrm{pH}$ and adaptation on high starch diets. Aus. J. agr. Res., 8, 691-710.

ROBINSON D. S., 1970 . The function of the plasma triglycerides in fatty acid transport. Comp. Biochem., 18, 51-116.

RONCARI D. A., MURTHY V. K., 1975. Effects of thyroid hormones on enzymes involved in fatty acid and glycerolipid synthesis. Evidence for tissue specificity. J. biol. Chem., 250, 4134-4138.

ROUS S., 1971. Rôle modeste du citrate comme transporteur d'acétyl CoA chez l'animal vivant. FEBS Leffers, 12, 338-340.

ROUS S., 1974. Différences de nature dans les systèmes de transport extra-miłochondrial des précurseurs de la synthèse des acides gras chez le rat et la souris. Biochimie, 56, 153-159.

ROUS S., 19760. Relative importance of acetate, acelo-acetate and D- $\beta-O H$-butyrate in the transport of acetyl COA from the mitochondria to the cytoplasm for fatty acid synthesis in mice. Life Sc., 18, 633-638.

ROUS S., 1976b. On the occurence of enzymes of ketone-body metabolism in human adipose tissue. Biochem. biophys. Res. Commun., 69, 74-78.

ROUS S., 1977. Fasting and insulin regulation of the utilization of aceto-acetate for fatty acid synthesis. Arch. Biochem. Biophys., 179, 328-333.

ROUS S., FAVARGER P., 1973. The role of aceto-acetate in the transfer of acetyl units outside the mitochondria in liver and adipose tissue of rats or mice. FEBS Letfers, 37, 231-233. 
RUDMAN D., DI GIROLAMO M., 1967. Comparative studies on the physiology of adipose tissue. Adv. Lipid Res., 5, 35-117.

SAITO T., TOMITA K., 1973. Two types of soluble malic enzyme in rat tissues. J. Biochem., Tokyo, 73, 803-810.

SCHOTZ M. C., GARFINKEL A. S., 1972. Effect of nutrition on species of lipoprotein lipase. Biochim. biophys. Acta., 270, 472-478.

SCOW R. O., HAMOSH M., BLANCHETTE-MACKIE E. J., EVANS A. J., 1972. Uptake of blood triglyceride by various tissues. Lipids, 7, 497-505.

SEUBERT W., SCHONER W., 1971. The regulation of pyruvate kinase. Curr. Top. Cell Reg., 3, 237. 267.

SEVERSON D. L., DENTON R. M., BRIDGES B. J., RANDLE P. J., 1976. Exchangeable and total calcium pools in mi tochondria of rat epididymal fat pads and isolated fatcells. Biochem J. 154, 209-223.

SICA V., CUATRECASAS P., 1973. Effects of insulin, epinephrine, and cyclic adenosine monophosphate on pyruvate dehydrogenase of adipose tissue. Biochemistry, 12, 2282-2291.

STANSBIE D., DENTON R. M., BRIDGES B. J., PASK H. T., RANDLE P. J., 1976. Regulation of pyruvate dehydrogenase and pyruvate dehydrogenase phosphatase activity in rat epididymal fat-pads. Biochem. J., 154, 225-236.

STEINBERG D., 1972. In W. L. HOLMES, R. PAOLETTI, D. KRITCHEVSKY. Pharmacological control of lipid Metabolism, p. 77, Plenum Press, New York.

SZEPESI B., FREEDLAND R. A., 1968. Alterations in the activities of several rat liver enzymes at various times after the feeding of high carbohydrate diets to rats previously adapted to a high protein regimen. J. Nutr., 94, 37-46.

THOMPSON W. J., WILLIAMS R. H., LITTLE S. A., 1973. Activation of guanyl cyclase and adenyl cyclase by secretin. Biochim. biophys. Acto, 302, 329-337.

TORNQVIST H., BELFRAGE P., 1976. Purification and some properties of a monoacyl glycerol hydrolyzing enzyme of rat adipose tissue. J. biol. Chem., 251, 813-819.

TURTLE J. R., KIPNIS D. M., 1967. An adrenergic receptor mechanism for the control of cyclic 3' 5' adenosine monophosphate synthesis in tissues. Biochem. biophys. Res. Commun., 28, 797.802.

VAUGHAN M., STEINBERG D., 1963. Effect of hormones on lipolysis and esterification of free fatty acids during incubation of adipose tissue in vitro. J. Lipid Res., 4, 193-199.

VAUGHAN M., STEINBERG D., 1965. In J. FIELD. Handbook of Physiology (Amer Physiol. Soc.) Sect. 5, p. 239, Williams and Wilkins, Baltimore, Maryland.

VAUGHAN M., 1972. In Insulin Action, p. 297, Acad. Press, New York/London.

VEECH R. L., GUYNN R. W., 1974. In Regulation of hepatic metabolism, p. 337, Munksgaard, Copenhagen.

VOLPE J. J., VAGELOS P. R., 1973a. Fatty acid synthetase of mammalian brain, liver and adipose tissue. Regulation by prosthetic group furnover. Biochim. biophys. Acta, 326, 293-304.

VOLPE J. J., VAGELOS P. R., 1973b. Satured fatty acid biosynthesis and its regulation. Ann. Rev. Biochem., 42, 21-60.

VOLPE J. J., MARASA J. C., 1975. Hormonal regulation of fatty acid synthetase, acetyl CoA carboxylase and fatty acid synthesis in mammalian adipose tissue and liver. Biochim. biophys. Acta. $380,454-472$.

WAKIL S. J. TITCHENER E. B., GIBSON D. M., 1958 . Evidence for the participation of biotin in the enzymic synthesis of fatty acids. Biochim Biophys. Acta, 29, 225-226.

WALSH D. A., ASHBY C. D., GONZALES C., CALKINS D., FISHER E. H., KREBS E. G., 1971. Purification and characterization of a protein inhibitor of adenosine $3^{\prime}, 5^{\prime}$-monophosphate dependent protein kinases. J. biol. Chem., 246, 1977-1985.

WIELAND O. H., PETZELT C., LÖFFLER G., 1972. Active and inactive forms of pyruvate deshydrogenase in rat liver. Eur. J. Biochem., 26, 426-433.

WILEY J. H., LEVEILLE G. A., 1973. Metabolic consequences of dietary medium chain triglycerides in the rat. J. Nutr., 103, 829-836.

WISE E. M., BALL E. G., 1964. Malic enzyme and lipogenesis. Proc. nat. Acad. Sci., USA, 52, 1255-1262.

WOLF C., VANHOVE A., BRETON M., ETIENNE J., BEREZIAT G., POLONOVSKI J., 1975. Localisation subcellulaire de la lipoprotéine lipase dans l'adipocyte de rat. C. R. Soc. Biol., 169, $1145-1149$. 